\title{
ATLAS and CMS measurements on spectroscopy
}

\author{
Jhovanny Andres Mejia Guisao*t \\ on behalf of the ATLAS and CMS Collaborations \\ Physics Institute, UNIVERSIDAD DE ANTIOQUIA, Medellin, Colombia. \\ E-mail: jhovanny.andres.mejia.guisaodcern.ch
}

\begin{abstract}
This proceeding contribution presents a selected number of studies performed by the ATLAS and the CMS experiments on b-hadron spectroscopy. Both collaborations have a rich b-hadron physics program on exploiting the large cross section of b-hadrons at the high energies of the LHC. For the ATLAS collaboration, we report the observation of an excited $B_{c}^{+}$meson state and the search for a structure in the $B_{s}^{0} \pi^{ \pm}$invariant mass spectrum. For the CMS collaboration, recent results in study of the $B^{+} \rightarrow J / \psi \Lambda p$ decay and observation of two excited $B_{c}^{+}$states and measurement of the $B_{c}^{+}(2 S)$ mass.
\end{abstract}

7th Annual Conference on Large Hadron Collider Physics - LHCP2019

20-25 May, 2019

Puebla, Mexico

\footnotetext{
* Speaker.

${ }^{\dagger}$ The author wants to acknowledge the financial support of COLCIENCIAS to attend to the conference.
} 


\section{Introduction}

The spectroscopy for hadrons with $\mathrm{b}$ quarks has been an essential experimental tool to understand QCD, and for this reason, several experiments at CERN and Fermilab had included these as an important part of their programs. With the full Run 2 of the LHC, the b-hadron spectroscopy at energies never reached before provides a new input to test theoretical calculations. Besides, the large statistics gives us the opportunity to look for new and exotic decay channels.

A wide program of studies of heavy flavor spectroscopy at the LHC is performed by the ATLAS [1] and CMS [2] collaborations and a number of recent results in this field are reviewed in this paper. However, several other contributions in this conference include some of the new results not covered here.

\section{Study of excited $B_{c}^{+}$mesons decays to $B_{c}^{+} \pi^{+} \pi^{-}$}

The states of the $B_{c}^{+}$meson family are unique in the Standard Model, consisting of charged mesons composed by a beauty quark and a charm antiquark (or vice versa). Although the ground state was discovered in 1998 by the CDF Collaboration [3], only at the LHC it became possible to perform a wide range of studies of its characteristics and searches for excited states, providing an excellent test ground for the existing theoretical calculations. The spectrum of this heavy quarkonium family is predicted to be very populated, but spectroscopic observations and measurements of production properties remain scarce. Indeed, their production yields are significantly smaller than those of the charmonium and bottomonium states, the $\bar{b} c$ production cross sections being proportional to the fourth power of the strong coupling constant, $\alpha^{4}$ (since two pairs of heavy quarks need to be produced). While the masses and sizes of these beauty-charm quark-antiquark pairs place them between the charmonium and bottomonium systems, so that many properties can be theoretically inferred by interpolation of existing knowledge, the unequal quark masses and velocities could lead to more complex dynamics, where some (nonrelativistic) approximations might break down. Since the $\bar{b} c$ mesons can not annihilate into gluons, the excited states decay to the ground state via the cascade emission of photons or pion pairs, leading to total widths that are less than a few hundred $\mathrm{KeV}$.

Both experiments, ATLAS and CMS, have studied excited states of $B_{c}^{+}$meson based mainly on the reconstruction of $B_{c}^{+} \pi^{+} \pi^{-}$decays with the $B_{c}^{+}$being reconstructed in the $B_{c}^{+} \rightarrow J / \psi \pi^{+}$ decay. Next subsections report the results from ATLAS and CMS respectively.

\subsection{Observation of an Excited $B_{c}^{+}$meson state with the ATLAS Detector}

The first observation of an excited state of $B_{c}^{+}$meson was reported [4] using $4.9 \mathrm{fb}^{-1}$ of $7 \mathrm{TeV}$ and $19.2 \mathrm{fb}^{-1}$ of $8 \mathrm{TeV}$ pp collision data collected by the ATLAS experiment at the LHC. The state appears in the distribution of the mass difference $Q=m\left(B_{c}^{+} \pi \pi\right)-m\left(B_{c}^{+}\right)-m(2 \pi)$. Figure 1 shows this distribution for 7 and $8 \mathrm{TeV}$ data with clear signal peaks visible in both.

The mass of the observed state is $6842 \pm 4$ (stat) \pm 5 (syst) $\mathrm{MeV}$, where the first error is statistical and the second is systematic, and it is consistent with the expectations for second S-wave excitations of the $\bar{b} c$ family, $B_{c}^{+}(2 S)$ and $B_{c}^{*+}(2 S)$. Therefore, the signal can be attributed either to the decay $B_{c}^{+}(2 S) \rightarrow B_{c}^{+} \pi^{+} \pi^{-}$or the decay $B_{c}^{*+}(2 S) \rightarrow B_{c}^{*+} \pi^{+} \pi^{-}$with a subsequent transition 

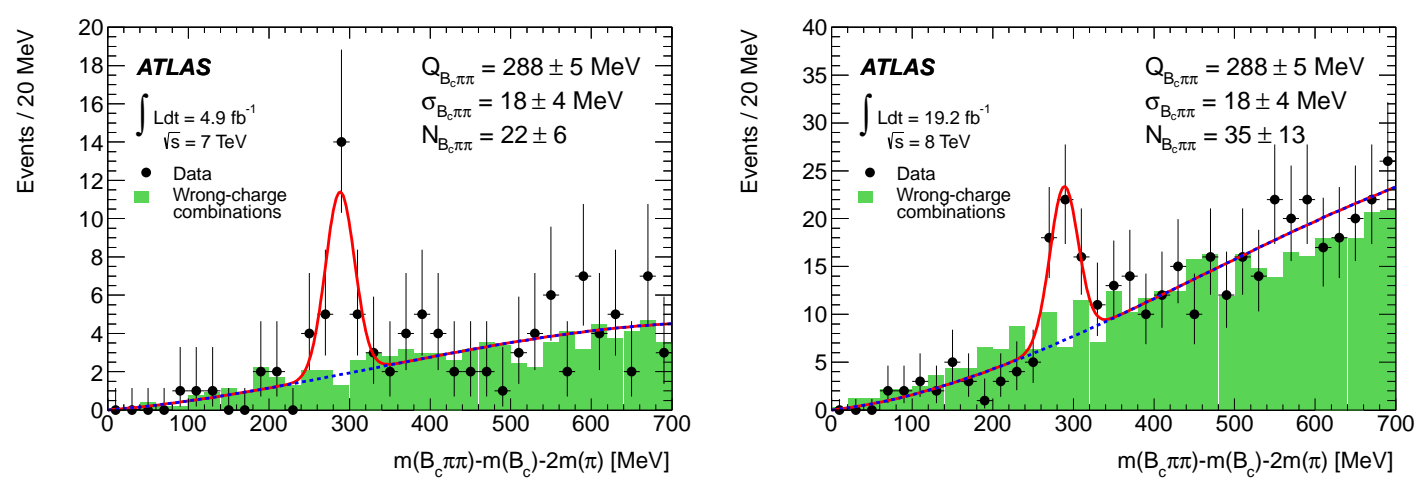

Figure 1: From Ref [4]. The $Q=m\left(B_{c}^{+} \pi \pi\right)-m\left(B_{c}^{+}\right)-m(2 \pi)$ distribution for the right-charge combinations (points with error bars) and for the same (wrong) pion charge combinations (shaded histogram) in 7 $\mathrm{TeV}$ (left) and $8 \mathrm{TeV}$ (right). The wrong-charge combinations are normalized to the same yield as the rightcharge background. The solid line is the projection of the results of the unbinned maximum likelihood fit to all candidates in the range $0-700 \mathrm{MeV}$. The dashed line is the projection of the background component of the same fit.

$B_{c}^{*+} \rightarrow B_{c}^{+} \gamma$ where the photon is not reconstructed, or the peak could be the superposition of the $B_{c}^{+}(2 S)$ and $B_{c}^{*+}(2 S)$ states, too closely spaced with respect to the resolution of the measurement.

Later, the LHCb Collaboration reported [5] that their $8 \mathrm{TeV}$ data sample did not show any significant sign of the $B_{c}^{+}(2 S)$ and $B_{c}^{*+}(2 S)$ states. Furthermore, upper limits set by the LHCb experiment on the relative $B_{c}^{+}(2 S)$ and $B_{c}^{*+}(2 S)$ production rate appear to be in apparent tension with the ATLAS Collaboration result, although at that moment it was not possible to make a definite conclusion on the consistency. That tension clearly motivated further studies by the experiments, in particular searches for these excitations in the full Run-2 data.

\subsection{Observation of two excited $B_{c}^{+}$states and measurement of the $B_{c}^{+}(2 S)$ mass in pp collisions at $13 \mathrm{TeV}$}

Signals consistent with the $B_{c}^{+}(2 S)$ and $B_{c}^{*+}(2 S)$ states have been separately observed for the first time [6] by investigating the $B_{c}^{+} \pi^{+} \pi^{-}$invariant mass spectrum measured by CMS. The analysis is based on the entire LHC sample of proton-proton collisions at a center-of-mass energy of $13 \mathrm{TeV}$, corresponding to a total integrated luminosity of $143 \mathrm{fb}^{-1}$. Figure 2 shows the final $M\left(B_{c}^{+} \pi^{+} \pi^{-}\right)-M\left(B_{c}^{+}\right)+m_{B_{c}^{+}}$mass distribution along with the result of an extended unbinned maximum likelihood fit, where $M\left(B_{c}^{+} \pi^{+} \pi^{-}\right)$and $M\left(B_{c}^{+}\right)$are, respectively, the reconstructed invariant masses of the $B_{c}^{+} \pi^{+} \pi^{-}$and $B_{c}^{+}$candidates, and $m_{B_{c}^{+}}$is the world-average $B_{c}^{+}$mass [7]. The two states are reconstructed as two well-resolved peaks, with a measured mass difference $29.1 \pm 1.5$ (stat) \pm 0.7 (syst) MeV. The observation of two peaks, rather than one, is established with a significance exceeding five standard deviations. The mass of the $B_{c}^{+}(2 S)$ meson is measured to be $6871.0 \pm 1.2$ (stat) \pm 0.5 (syst) $\mathrm{MeV}$, where the last term corresponds to the uncertainty in the world-average $B_{c}^{+}$mass. Since the low-energy photon emitted in the $B_{c}^{+} \gamma$ radiative decay is not reconstructed, the observed $B_{c}^{*+}(2 S)$ peak has a mass lower than the true value, which remains unknown. 


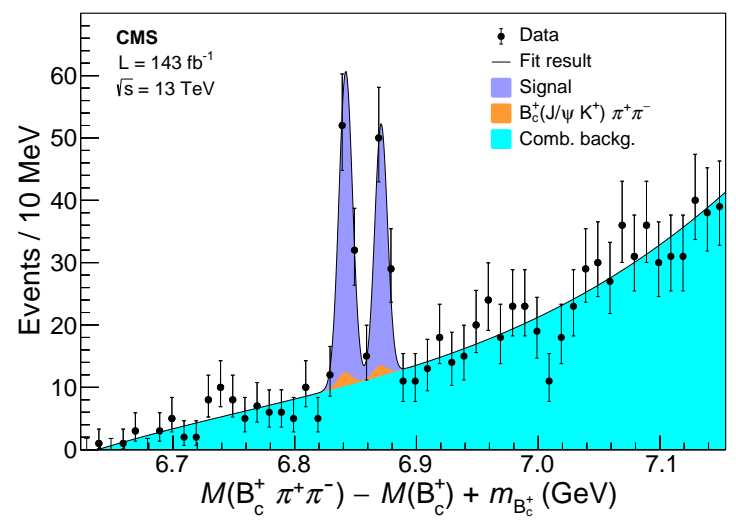

Figure 2: From Ref [6]. The $M\left(B_{c}^{+} \pi^{+} \pi^{-}\right)-M\left(B_{c}^{+}\right)+m_{B_{c}^{+}}$distribution. The $B_{c}^{+}(2 S)$ is assumed to be the right-most peak. The vertical bars on the points represent the statistical uncertainty in the data. The contributions from the various sources are shown by the stacked distributions. The solid line represents the result of the fit.

Recently, LHCb collaboration presented an updated search for excited $B_{c}^{+}$mesons in the $B_{c}^{+} \pi^{+} \pi^{-}$mass distribution [8], and confirmed the two peaks observed by the CMS experiment.

\section{Search for a structure in the $B_{s}^{0} \pi^{ \pm}$invariant mass spectrum with the ATLAS Experiment}

The evidence of a new narrow structure, $X(5668)$, in the decay $X(5668) \rightarrow B_{s}^{0} \pi^{ \pm}$reported by the $\mathrm{D} \varnothing$ Collaboration $[9,10]$ awakened a lot of interest within the community that studies exotic hadrons [11] and triggered this search at several hadron collider experiments, including ATLAS [12], CMS [13], LHCb [14] and CDF [15].

The search for the $X(5668)$ state by the ATLAS experiment at the LHC, by studying resonancelike structures in the $B_{s}^{0} \pi^{ \pm}$invariant mass spectrum was performed based on a combined sample of pp collision data at $\sqrt{s}=7 \mathrm{TeV}$ and $\sqrt{s}=8 \mathrm{TeV}$ corresponding to integrated luminosities of 4.9 and $19.5 \mathrm{fb}^{-1}$, respectively. The $B_{s}^{0}$ candidates are reconstructed in the decay chain $B_{s}^{0} \rightarrow J / \psi \phi$, $J / \psi \rightarrow \mu^{+} \mu^{-}, \phi \rightarrow K^{+} K^{-}$. The $B_{s}^{0} \pi^{ \pm}$invariant mass distributions do not show any unexpected structures. The results of the fits are shown in Figure 3. Within the acceptance in which this analysis is performed, upper limits on the number of signal events, $(\mathrm{N}(\mathrm{X}))$, and on the $X$ production rate relative to $B_{s}^{0}$ mesons $\left(\rho_{X}\right)$, were determined at $95 \%$ C.L., resulting in $N(X)<382$ and $\rho_{X}<0.015$ for $p_{T}\left(B_{s}^{0}\right)>10 \mathrm{GeV}$, and $N(X)<356$ and $\rho_{X}<0.016$ for $p_{T}\left(B_{s}^{0}\right)>10 \mathrm{GeV}$. Limits are also set for potential $B_{s}^{0} \pi^{ \pm}$resonances in the mass range 5550 to $5700 \mathrm{MeV}$. The results are shown in Figure 4.

\section{Study of the $B^{+} \rightarrow J / \psi \Lambda p$ decay in proton-proton collisions at $8 \mathrm{TeV}$}

Recently, the LHCb experiment reported the observation of structures in the $J / \psi p$ mass spectrum from $\Lambda_{b}^{0} \rightarrow J / \psi K p$ decays [16, 17]. A natural extension of these searches is the study of the mass spectra in other b-hadrons channels like $B^{+} \rightarrow J / \psi \Lambda p$. The CMS experiment performed 

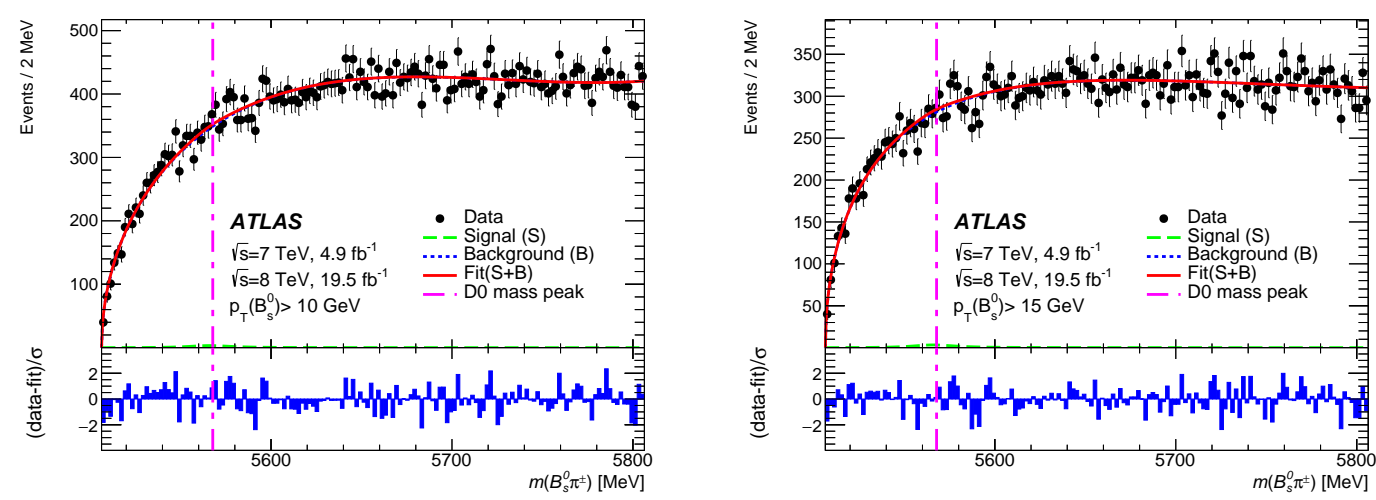

Figure 3: From Ref [12]. Results of the fit to the $B_{s}^{0} \pi^{ \pm}$mass distribution for candidates with $p_{T}\left(B_{s}^{0} \pi\right)>10$ $\mathrm{GeV}$ (left) and $p_{T}\left(B_{s}^{0} \pi\right)>15 \mathrm{GeV}$ (right). The bottom panels show the difference between each data point and the fit divided by the statistical uncertainty of that point.

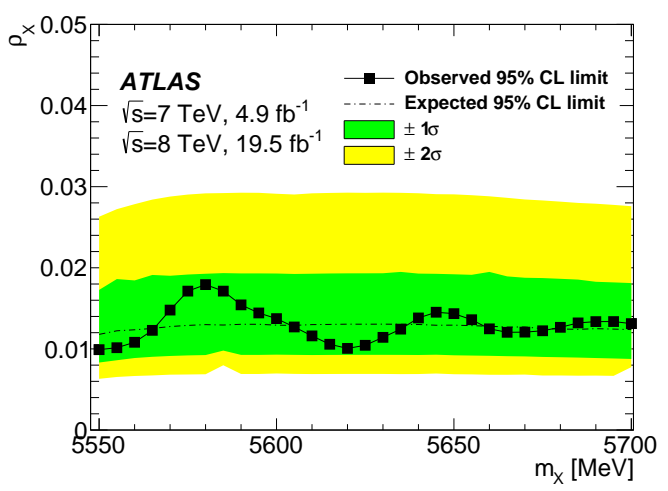

Figure 4: From Ref [12]. Upper limits on $\rho_{X}$ at 95\% CL (black squares connected by line) at different masses of a hypothetical resonant state $\mathrm{X}$ decaying to $B_{s}^{0} \pi^{ \pm}$, for events with $p_{T}\left(B_{s}^{0} \pi\right)>10 \mathrm{GeV}$. A Breit Wigner width of $21.9 \pm 6.4$ (stat) ${ }_{-2.5}^{+5.0}$ (syst) $\mathrm{MeV}$ is assumed, as reported by $\mathrm{D} \varnothing$. The values include systematic uncertainties. The expected $95 \%$ CL upper limits (central black dot-dashed line) with $\pm 1 \sigma$ (green) and $\pm 2 \sigma$ (yellow) uncertainty bands on $\rho_{X}$ are shown as a function of the assumed resonance mass.

such studies and reported [18] $]^{1}$ the branching fraction measurement of $B^{+} \rightarrow J / \psi \Lambda p$, which is the most precise to date and consistent with the previous Belle measurement [20].

Using the data set of proton-proton collisions, collected by the CMS experiment at $\sqrt{s}=$ $8 \mathrm{TeV}$ and corresponding to an integrated luminosity of $19.6 \mathrm{fb}^{-1}$, the branching fraction ratio $\frac{\beta\left(B^{+} \rightarrow J / \psi \Lambda p\right)}{\beta\left(B^{+} \rightarrow J / \psi K^{*+}\right)}=(1.054 \pm 0.087$ (stat) \pm 0.028 (syst) $\pm 0.011(\beta)) \times 10^{-2}$ was measured. Using the world-average branching fraction of the $B \rightarrow J / \psi K^{*+}$ decay, it can be obtained $\beta(B \rightarrow J / \psi \Lambda p)=$ $(15.07 \pm 0.81$ (stat) \pm 0.40 (syst) $\pm 0.86(\beta)) \times 10^{-6}$. The invariant mass distribution of the selected $B^{+} \rightarrow J / \psi \Lambda p$ candidates is shown in Figure 5. The study of two-body invariant mass distributions of the $B^{+} \rightarrow J / \psi \Lambda p$ decay products was performed, showing that the spectra can not be satisfactory modeled with a phase space distribution. Figure 6 shows the invariant mass distributions of the $J / \psi p, J / \psi \Lambda$ and $\Lambda p$ systems. A model-independent approach was used to conclude that the

\footnotetext{
${ }^{1}$ The preliminary results shown in this contribution are superseded in this paper [19], submitted to JHEP.
} 
agreement is improved significantly, once the contribution from $K^{*}$ resonances with spins up to 4 in the $\Lambda p$ system is accounted for.

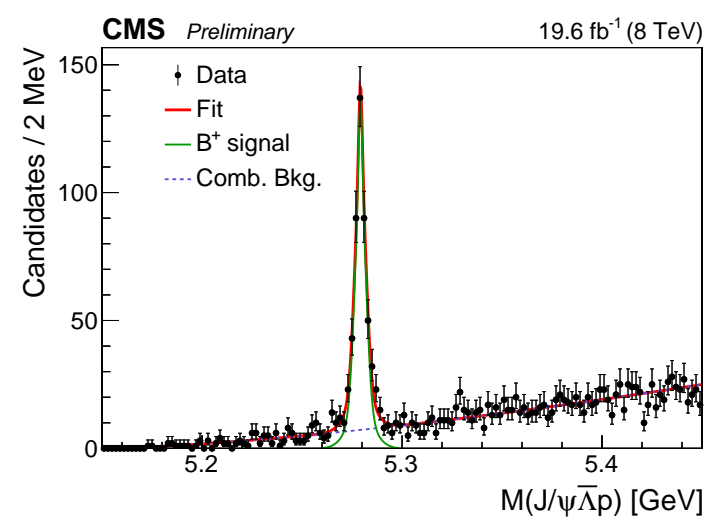

Figure 5: From Ref [18]. Invariant mass distribution of the selected $B^{+} \rightarrow J / \psi \Lambda p$ candidates. The points are data and the curves are results of the fits.

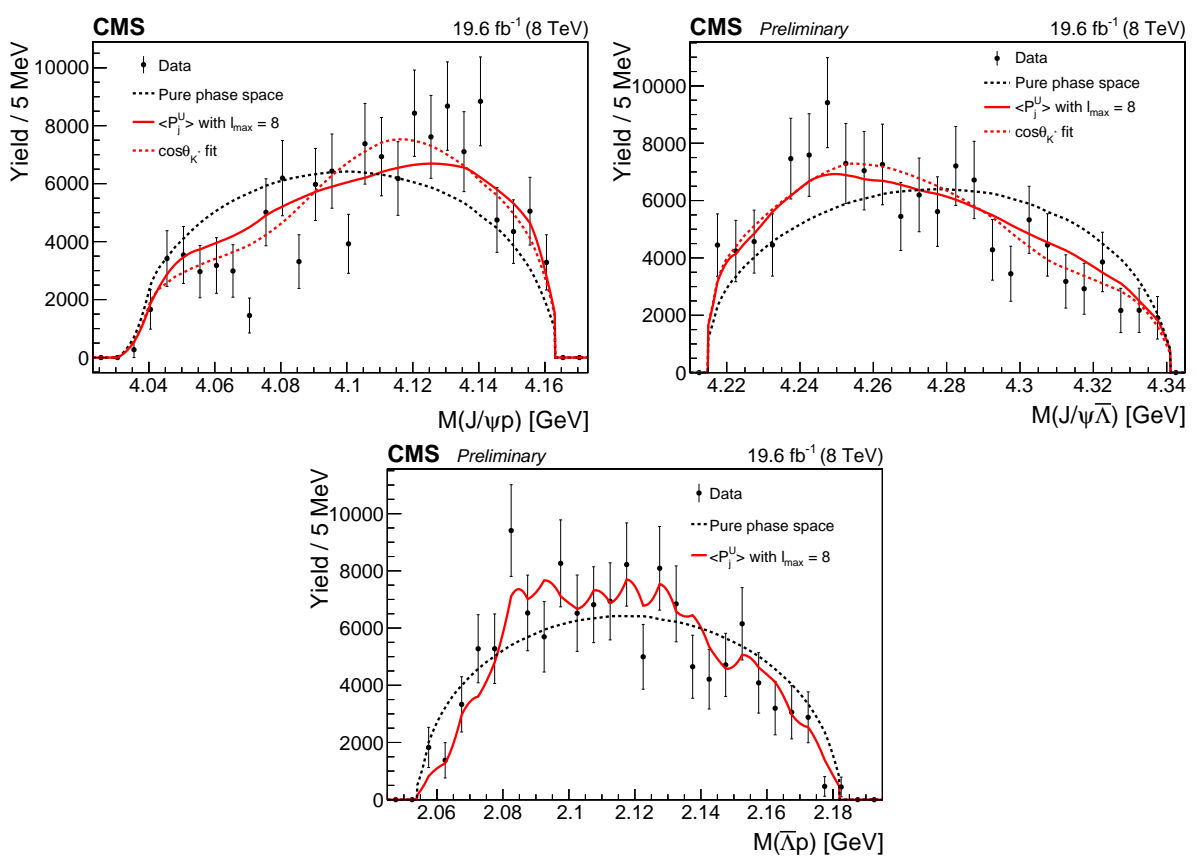

Figure 6: From Ref [18]. The invariant mass distributions of the $J / \psi p$ (upper left), $J / \psi \Lambda$ (upper right), and $\Lambda p$ (lower) systems from the $B^{+} \rightarrow J / \psi \Lambda p$ decay. The points are efficiency-corrected and backgroundsubtracted data. Superimposed curves are obtained from simulation: the red curve represents the phase space distribution corrected by the $\Lambda p$ angular structure with the inclusion of the first eight moments corresponding to the resonances in the $\Lambda p$ system; the dashed red curve is the fit to the phase space distribution reweighted. 


\section{References}

[1] G. Aad et al. ATLAS Collaboration, "The ATLAS Experiment at the CERN Large Hadron Collider", JINST, 3, S08003 (2008).

[2] S. Chatrchyan et al. CMS Collaboration, "The CMS Experiment at the CERN LHC", JINST 3, S08004 (2008).

[3] F. Abe et al, CDF Collaboration, "Observation of the $B_{c}^{+}$Meson in $P \bar{P}$ Collisions at $\left.\sqrt{(} s\right)=1.8$ TeV", Phys. Rev. Lett. 81, 2432 (1998).

[4] G. Aad et al. ATLAS Collaboration, "Observation of an Excited $B_{c}^{+}$meson state with the ATLAS Detector", Phys. Rev. Lett. 113, 212004 (2014).

[5] R. Aaij et al, LHCb collaboration, "Search for excited $B_{c}^{+}$states", J. High Energ. Phys. (2018) 2018: 138.

[6] G. Aad et al. CMS Collaboration, "Observation of two excited $B_{c}^{+}$states and measurement of the $B_{c}^{+}(2 S)$ mass in pp collisions at $\left.\sqrt{(} s\right)=13$ TeV", Phys. Rev. Lett. 122, 132001 (2019).

[7] M. Tanabashi et al. (Particle Data Group), Review of particle physics, Phys. Rev. D 98, 030001 (2018).

[8] R. Aaij et al, LHCb collaboration, "Observation of an Excited $B_{c}^{+}$state", Phys. Rev. Lett. 122, 232001 (2019).

[9] V. M. Abazov et al, D $\varnothing$ Collaboration, "Evidence for a $B_{s}^{0} \pi^{ \pm}$State", Phys. Rev. Lett. 117, 022003 (2016).

[10] V. M. Abazov et al, D $\varnothing$ Collaboration, "Study of the $X(5568)$ state with semileptonic decays of the $B s^{0}$ meson", Phys. Rev. D 97, 092004 (2018)

[11] Feng-Kun Guo, Ulf-G. Mei $\beta$ ner, and Bing-Song Zou, "How the $X(5568)$ challenges our understanding of QCD", Commun. Theor. Phys., 65(5):593-595,2016.

[12] M. Aaboud et al, ATLAS Collaboration, "Search for a structure in the $B_{s}^{0} \pi^{ \pm}$invariant mass spectrum with the ATLAS Experiment", Phys. Rev. Lett. 120, 202007 (2018).

[13] A. M. Sirunyan et al, CMS Collaboration, "Search for the $X(5568)$ State Decaying into $B_{s}^{0} \pi^{ \pm}$in Proton-Proton Collisions at $\sqrt{(s)}=8$ TeV" Phys. Rev. Lett. 120, 202005 (2018).

[14] R. Aaij et al, LHCb Collaboration, "Search for Structure in the $B_{s}^{0} \pi^{ \pm}$Invariant Mass Spectrum, Phys. Rev. Lett. 117, 152003 (2016).

[15] T. Aaltonen et al, CDF Collaboration, "Search for the Exotic Meson X(5568) with the Collider Detector at Fermilab", Phys. Rev. Lett. 120, 202006 (2018).

[16] R. Aaij et al,LHCb Collaboration, "Observation of $J / \psi p$ resonances consistent with pentaquark states in $\Lambda_{b}^{0} \rightarrow J / \psi K p$ decays", Phys. Rev. Lett. 115, 072001 (2015).

[17] R. Aaij et al,LHCb Collaboration, "Observation of a narrow pentaquark state, $P_{c}(4312)^{+}$, and of the two-peak structure of the $P_{c}(4450)^{+"}$, Phys. Rev. Lett. 122, 222001 (2019).

[18] CMS Collaboration, "Study of the $B^{+} \rightarrow J / \psi \Lambda p$ decay in proton-proton collisions at $\sqrt{(s)}=8 \mathrm{TeV}$ ", CMS-PAS-BPH-18-005.

[19] CMS Collaboration, "Study of the $B^{+} \rightarrow J / \psi \Lambda p$ decay in proton-proton collisions at $\sqrt{(s)}=8 \mathrm{TeV}$ ", arXiv:1907.05461[hep-ex].

[20] Q. L. Xie et al, Belle Collaboration, "Observation of $B^{+} \rightarrow J / \psi \Lambda p$ and searches for $B^{+} \rightarrow J / \psi \Sigma P$ and $B^{0} \rightarrow J / \psi P P^{\prime}$, Phys. Rev. D 72, 051105(R) (2005). 